\title{
Short-term and long-term collaboration benefits on individual recall in younger and older adults
}

\author{
Helena M. Blumen • Yaakov Stern
}

Published online: 2 November 2010

(C) The Psychonomic Society 2010

\begin{abstract}
A recent study of younger adults suggests that, compared to repeated individual recall trials, repeated collaborative recall trials produce better individual recall after a short delay (Blumen \& Rajaram, 2008). Our study was designed to determine if such collaboration benefits would remain after a one-week delay, in both younger and older adults. Sixty younger $(M$ age $=24.60)$ and 60 older $(M$ age $=67.35)$ adults studied a list of words and then completed either two collaborative recall trials followed by two individual recall trials, or four individual recall trials. A five-min delay was inserted between the first three recall trials. The fourth recall trial was administered 1 week later. Collaborative recall was completed in groups of three individuals working together. Both younger and older adults benefitted from repeated collaborative recall trials to a greater extent than repeated individual recall trials, and such collaboration benefits remained after a one-week delay. This is the first demonstration of collaboration benefits on later individual recall at delays as long as 1 week, in both younger and older adults. Findings are discussed within the context of the negative effects of collaboration associated with group memory (collaborative inhibition) and the positive effects of collaboration associated with later individual memory (collaboration benefits).
\end{abstract}

Keywords Collaborative memory - Group memory · Collaborative inhibition - Collaboration benefits .

Re-exposure $\cdot$ Cross-cuing

H. M. Blumen $(\bowtie) \cdot$ Y. Stern

Columbia University, Sergievsky Center,

P\&S Box 16, 630 W. 168th Street,

New York, NY 10032, USA

e-mail: hmb2131@columbia.edu

\section{Introduction}

Younger and older adults frequently discuss or recall information in a social context. Younger adults attend study groups to prepare for upcoming exams and older adults reside in group settings where they frequently share day-today experiences. Yet, very little is known about how such group collaboration modulates later individual memory, particularly in older adults. In younger adults, repeated collaborative recall trials have been shown to produce better individual recall than repeated individual recall trials, after short delay (Blumen \& Rajaram, 2008). Our study was designed to (1) replicate this finding in younger adults, (2) extend this finding to older adults, and (3) determine if such collaboration benefits would remain after a one-week delay in both younger and older adults.

Collaborative memory research to date has focused on the negative effects of collaboration associated with group memory. In a typical collaborative memory study, individuals are presented with a list of words and shortly thereafter they are asked to recall these words either individually, or in a group of two (or three) individuals working together. Collaborative group recall is then compared to nominal group recall that is obtained by pooling the nonoverlapping recall of an equal number of individuals working alone. The key finding in this paradigm is counterintuitive: collaborative group recall is lower than nominal group recall. This counterintuitive finding has been termed collaborative inhibition (Weldon \& Bellinger, 1997) and occurs to a similar extent in younger and older adults (Ross, Spencer, Blatz \& Restorick, 2008; Meade \& Roediger, 2009).

Collaborative inhibition is typically considered to be a function of retrieval disruption (Basden, Basden, Bryner \& Thomas, 1997; Weldon \& Bellinger, 1997). The retrieval 
disruption account maintains that responses provided by one of the group members disrupts the retrieval organization of the other group members and prevent them from contributing some of the information that they would have contributed if they were asked to recall individually. The retrieval disruption account also maintains that disruption is temporary and should not influence later individual memory, even after a short delay. Indeed, there is some evidence to suggest that the information that is lost during collaborative recall is largely recovered during later individual recall assessed after a short (3-min) delay, providing strong support for this proposition (Finlay, Hitch \& Meudell, 2000). Such recovery presumably occurs because individuals can return to their individual retrieval organization during later individual recall. In our study, greater recovery shortly $(5 \mathrm{~min})$ following repeated collaborative recall trials than repeated individual recall trials, would provide additional support for this proposition.

Collaborative inhibition can also be considered to be a function of socially-shared retrieval-induced forgetting (SS-RIF; Coman, Manier, \& Hirst, 2009; Cuc, Koppel, \& Hirst, 2007; Stone, Barnier, Sutton \& Hirst, 2010). The SS-RIF account maintains that responses provided by one of the group members (cued information) are covertly retrieved by the other group members and lead to inhibition of non-cued information, i.e. the information that remains to be recalled. Unlike the typical collaborative memory study, an SS-RIF study involves an encoding phase, a collaborative cued recall phase and a subsequent individual free recall phase, and the presence or absence of collaborative inhibition during collaborative recall is not formally assessed. The collaborative retrieval phase involves cued rather than free recall, to control the information that is collaboratively retrieved and allow comparisons of later individual memory for information that was overtly retrieved $(\mathrm{Rp}+)$, covertly retrieved (Rp-) and not retrieved (Nrp) during collaboration. The key finding in this paradigm is also counterintuitive: while later individual recall for $\mathrm{Rp}+$ information is enhanced, later individual recall for $\mathrm{Rp}$ - information is reduced relative to Nrp information. Thus, the SS-RIF paradigm clearly shows that the negative effects associated with collaboration persist and lowers later individual memory as well. Note that SS-RIF has been observed up to $20 \mathrm{~min}$ following collaboration (Stone, Barnier, Sutton \& Hirst, 2010), but is unlikely to persist after a one-week delay. This is because the parallel phenomenon in the individual memory literature, within-individual retrieval-induced forgetting (Anderson, 2005; Anderson, Bjork \& Bjork, 1994), is eliminated after a long delay ( $24 \mathrm{~h})$ between the individual cued recall phase and later individual recall (MacLeod \& Macrae, 2001). In our study, greater recovery 1 week following repeated collaborative recall trials than repeated individual recall trials, would provide empirical support for this proposition.

Collaborative memory research to date has paid very little attention to the positive effects of collaboration associated with later individual memory. Collaboration can potentially benefit later individual memory through two different mechanisms: re-exposure and cross-cuing. When individuals recall information in a social context they are often re-exposed to additional information recalled by the other members of the group that they would not have recalled themselves (Weldon \& Bellinger, 1997; Blumen \& Rajaram, 2008). During collaborative recall, such reexposure benefits are obscured by the negative effects of collaboration, but can be detected during later individual recall. When individuals recall information in a social context the recall of others group members can also crosscue or trigger recall of new information that would not be available to them if recalling alone (Meudell, Hitch \& Boyle, 1995; Meudell, Hitch \& Kirby, 1992). Like reexposure benefits, cross-cuing benefits are obscured by the negative effects of collaboration, but can be detected during later individual recall.

Repeated attempts to obtain empirical evidence for the presence of cross-cuing during collaborative recall have been unsuccessful, despite clever designs (e.g. Meudell et al., 1992, 1995). Some empirical evidence for the presence of cross-cuing was recently obtained during repeated collaborative recall trials (Blumen \& Rajaram, 2008). In this study, repeated collaborative recall trials led to better individual recall after a short (5-min) delay than repeated individual recall trials (CCI > III), and a single collaborative recall trial followed by an individual recall trial $(\mathrm{CCI}>$ CII). Although the explanatory framework in this study centered on re-exposure, there was some evidence that cross-cuing contributed to these short-term collaboration benefits as well. This is because hypermnesia (the net increase in recall across recall trials, e.g. Payne, 1987) from the first recall trial to the second recall trial was greater in the repeated collaborative recall condition than in the repeated individual recall condition (CCI > III). Note that this increase in recall across repeated collaborative recall trials cannot be attributed to hypermnesia in general, because it is an increase that goes above and beyond that of repeated individual recall trials. More importantly, this increase in recall across repeated collaborative recall trials cannot be attributed to re-exposure, because no reexposures (or additional study opportunities) were provided between recall trials (Blumen \& Rajaram, 2008; Meudell et al., 1992, 1995; Weldon \& Bellinger, 1997).

Our study was not designed to isolate collaboration benefits generated by re-exposure and cross-cuing per se but utilized repeated collaborative recall trials because they have been shown to produce short-term collaboration 
benefits on later individual memory in younger adults. Such collaboration benefits are typically difficult to detect following a single collaborative recall trial in both younger (Blumen \& Rajaram, 2008; Finlay et al., 2000; Meudell et al., 1992, 1995; Wright \& Klumpp, 2004) and older adults (Meade \& Roediger, 2009; Ross et al., 2008).

Our study also utilized repeated collaborative recall trials because they potentially promote cross-cuing and crosscuing may be critical for detecting long-term collaboration benefits on later individual memory. This is because reexposure is more comparable to additional study opportunities, while cross-cuing is more comparable to additional retrieval opportunities. Hence, in the individual memory literature, additional study exposures have been shown to generate short-term benefits on later individual recall, while additional retrieval opportunities have been shown to be critical for long-term retention (e.g. Roediger \& Karpicke, 2006). In other words, while short-term collaboration benefits on later individual memory likely are generated by a combination of re-exposure and cross-cuing, long-term collaboration benefits on later individual memory are more likely the result of cross-cuing. Thus, our study utilized repeated collaborative recall trials to increase the possibility of detecting collaboration benefits on later individual recall after a long (1 week) delay. Given that older adults have weaker free recall performance than younger adults in general (e.g. Craik \& McDowd, 1987) they are possibly subject to less re-exposure during collaborative recall. Therefore, short-term collaboration benefits on later individual recall potentially will be greater in younger than older adults. Given that cross-cuing largely depends on reexposure, long-term collaboration benefit on later individual recall potentially will be greater in younger than older adults as well. However, cross-cuing also depends on an individual's ability to use the other group members' responses as retrieval cues during collaborative recall. Given that age-related differences in memory are reduced when retrieval cues are provided during recall (e.g. Craik, Byrd \& Swanson, 1987), it is possible that older adults are more likely than younger adults to use the other group members' responses as retrieval cues during collaborative recall. If this is the case, long-term collaboration benefits on later individual recall potentially will be greater or similar in older compared to younger adults, despite less reexposure.

\section{Methods \\ Participants}

A total of 60 younger $(M$ age $=24.60)$ and 60 older $(M$ age $=$ 67.35) adults participated in this study. Participants were recruited via fliers posted around Columbia University Medical Center. Older adults were screened for dementia with the Mattis' Dementia Rating Scale (DRS, Mattis, 1976) prior to inclusion in this study. A total DRS score of 135 or higher were required for inclusion. Additional demographic and screening information for each age group are provided in Table 1. Younger and older adults differed in terms of age and education (fewer older than younger adults had graduated from college), but not in terms gender (see Table 1). Participants provided written consent and were debriefed at the completion of the experiment.

\section{Design}

Age (Younger and Older) and type of retrieval sequence (CCI-I and III-I) were between-subjects factors and recall trial (Recall 1, Recall 2, Recall 3 and Recall 4) was a within-subjects factor. However, this overall design was reduced for the specific measures or questions of interest (see statistical approach). Ten three-person groups were randomly assigned to each retrieval sequence (i.e. a total of 30 younger and 30 older adults were assigned to each retrieval sequence condition).

\section{Materials}

Study items were composed of 54 concrete nouns (40 targets, 7 primacy buffers, 7 recency buffers; Clark \& Paivio, 2004). Two randomly ordered study lists were created to minimize order effects. Study items were presented on a computer screen but participant responses were recorded with a paper and pencil.

\section{Procedure}

After providing informed consent, participants completed a brief demographic questionnaire, followed by a study phase and an initial recall phase (Recall 1, Recall 2 and Recall 3). During the study phase, participants provided a pleasantness rating for the meaning of each study item on a scale from 1 to 5 (very unpleasant to very pleasant). Each study

Table 1 Means and standard deviations (in parentheses) of demographic and screening information for younger and older adults

\begin{tabular}{lll}
\hline Condition & Younger & Older \\
\hline Age* & $24.60(2.69)$ & $67.35(4.65)$ \\
\% Female & $0.72(0.45)$ & $0.63(0.49)$ \\
\% with college education* & $0.82(0.39)$ & $0.65(0.48)$ \\
DRS (Total) & N/A & $140.62(2.41)$ \\
\hline
\end{tabular}

$D R S$ dementia rating scale, $N / A$ not applicable

$* p<0.05$, two-tailed $t$-test 
item was presented for $6 \mathrm{~s}$ with a 2-s inter-stimulus interval (ISI). Upon completion of the study phase, participants completed a 7-min distracter task (recalling US cities). During the initial recall phase that followed, Recall 1 and Recall 2 were completed either individually or in groups of three individuals working together. Recall 3 was individually completed. During an individual recall trial, participants were asked to recall as many words as they could in any order. During a collaborative recall trial, participants were asked to collaboratively recall as many words as they could in any order. Participants were encouraged to contribute as many words as they could and to solve disagreements among themselves. One person was randomly asked to write the words down. Participants were given $7 \mathrm{~min}$ for recall (collaborative or individual) and a 5-min break was inserted between each recall trial. Upon completion of the initial retrieval phase, participants completed a brief post-experiment questionnaire (unrelated to the predictions of this study) and were told that they would be contacted in a week for some follow-up questions. No mention of a subsequent memory task was made. The delayed recall trial (Recall 4) was individually completed over the phone 7 to 10 days after the completion of the initial recall phase. During this delayed recall trial, participants were given $5 \mathrm{~min}$ to recall as many words as they could in any order. Participant responses were recorded by the experimenter. At the end of these $5 \mathrm{~min}$, the experimenter read the words that the participant had recalled to make sure they had been recorded correctly. Participants were then given an additional minute to recall any additional items.

\section{Results and discussion}

Statistical approach

Collaborative and individual recall performance was assessed in four different ways. First, the presence and absence of collaborative inhibition in younger and older adults during Recall 1 and Recall 2 was assessed by comparing collaborative group recall with nominal group recall. Nominal group recall was computed by pooling the non-redundant responses of three individuals working alone. The traditional ways to compute nominal group recall have been to randomly pool the responses of three individuals working alone or to pool the responses of three individuals that appear next to each other in a spreadsheet. However, as pointed out by others, such methods introduce unnecessary errors (Wright, 2007). The optimal way to calculate nominal group recall would be to generate all possible groupings of the data set. However, this approach would be computationally difficult even for modern day computers. Our study utilized an approach that was recently worked out and programmed by Kelley \&
Wright (2010) which falls somewhere in between these two alternatives. In short, their program generates 10,000 different ways to group the data and calculates a mean and standard deviation for this large set. Then, it runs a Chisquare test against this mean and standard deviation and provides the grouping that best fits with the mean for the large set. This grouping is then used in the inferential analyses. Second, collaboration benefits in younger and older adults after a shorter (Recall 3) and longer (Recall 4) delay were assessed by comparing individual recall following repeated collaborative recall trials to individual recall following repeated individual recall trials (CCI-I vs. III-I and CCI-I vs. III-I). Third, the presence of cross-cuing during repeated collaborative recall trials was assessed by comparing hypermnesia - the net increase in recall-from Recall 1 to Recall 2 in the collaborative condition and the individual condition (CCI-I vs. III-I). Finally, recovery (items recovered) was assessed from Recall 2 to Recall 3, and Recall 3 to Recall 4 to examine whether recovery would be greater shortly following collaborative than individual recall trials (indicating a recovery from retrieval disruption) and whether recovery would continue to be greater following collaborative than individual recall trials after a 1-week delay (indicating a release from SS-RIF).

\section{Collaborative inhibition}

The proportions of collaborative and nominal group recall during Recall 1 and Recall 2 in younger and older adults are displayed in Fig. 1. The presence and absence of

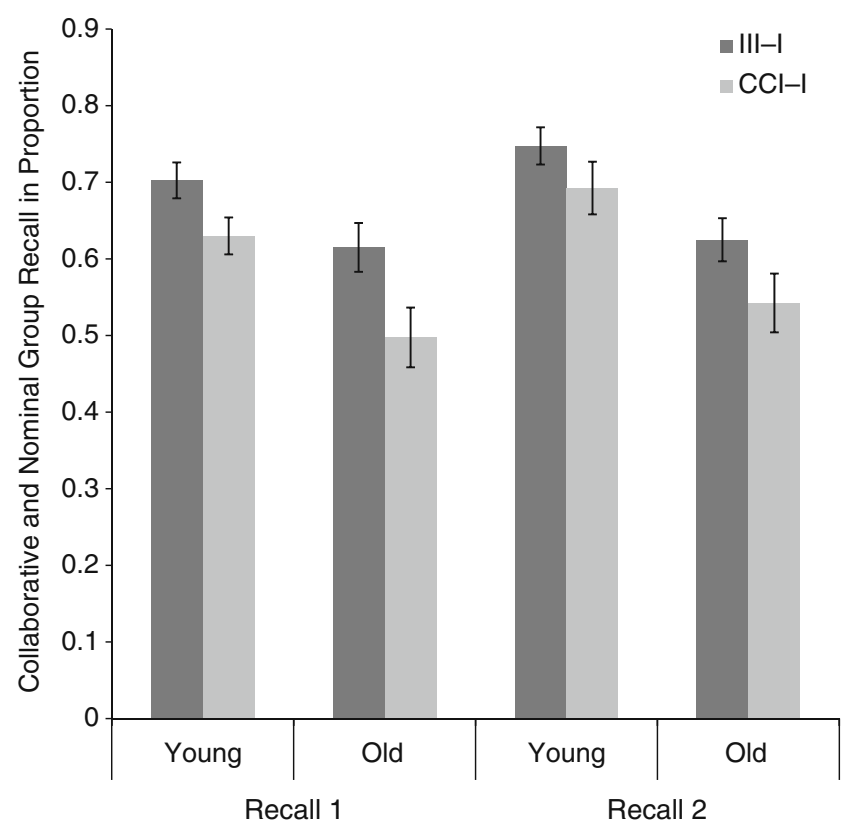

Fig. 1 Mean proportion of correct recall for nominal and collaborative groups in each age group during Recall 1 and Recall 2 
collaborative inhibition as a function of age and retrieval trial was assessed in a repeated measure ANOVA with recall trial (Recall 1 and Recall 2) as the within-subjects variable, and age (Younger and Older) and retrieval condition (Collaborative or Individual [Nominal]) as between-subjects variables. There was a main effect of recall trial, $F(1,36)=7.99, p<0.01, \eta p^{2}=0.18$, reflecting an increase in recall performance from Recall 1 to Recall 2. There was also a main effect of age, $F(1,36)=20.05, p<$ $0.001, \eta \mathrm{p}^{2}=0.36$, reflecting better recall performance in younger relative to older adults. Recall trial did not interact with age $F(1,36)=0.83, p>0.05$ or retrieval condition, $F$ $(1,36)=.83, p>.05$. More importantly (for the present purposes), there was a main effect of retrieval condition, $F$ $(1,36)=8.87, p<0.01, \eta p^{2}=0.20$, indicating the presence of collaborative inhibition, i.e., collaborative group recall was lower than nominal group recall. Although there was a numerical trend towards smaller collaborative inhibition in younger relative to older adults ( $7 \%$ versus $12 \%$ during Recall 1 and $6 \%$ versus $8 \%$ during Recall 2), the interaction between age and retrieval condition was not significant, $F$ $(1,36)=0.44, p>0.05$. The three-way interaction between retrieval trial, age and retrieval condition was also not significant, $F(1,36)=0.09, p>0.05$. These collaborative inhibition results show that levels of collaborative inhibition are similar in younger and older adults and do not attenuate during repeated retrieval trials. Similar levels of collaborative inhibition in younger and older adults nicely replicate previous research (Ross et al., 2008; Meade \& Roediger, 2009) and extend them to repeated collaborative recall trials.

\section{Individual recall}

The proportions of individual recall during Recall 3 and Recall 4 in younger and older adults are displayed in Fig. 2. Collaboration benefits on later individual recall as a function of age and retrieval trial was assessed in a repeated measure ANOVA with recall trial (Recall 3 and Recall 4) as the within-subjects variable, and age (Younger and Older) and retrieval condition (Collaborative or Individual) as between-subjects variables. There was a main effect of recall trial $F(1,116)=320.64, p<0.001, \eta p^{2}=0.73$, reflecting an overall decrease in recall performance after a 1-week delay. There was also a main effect of age, $F$ (1, $116)=68.79, p<0.001, \eta p^{2}=0.37$, reflecting better recall in younger than older adults. Recall trial did not interact with age $F(1,116)=2.52, p>0.05$, or retrieval condition, $F(1$, $116)=1.15, p>0.05$. More importantly, there was a main effect of retrieval condition, $F(1,116)=28.93, p<0.001$, $\eta p^{2}=0.20$, such that individual recall following repeated collaborative recall trials was better than following repeated individual recall trials. Although there was a numerical trend towards greater collaboration benefits on later individual recall in younger relative to older adults after a short delay (14\% versus $7 \%$ during Recall 3 ) and similar collaboration benefits on later individual recall in younger and older adults after a longer delay (9\% versus $8 \%$ during Recall 4 ), there was only a trend towards an interaction between age and retrieval condition, $F(1,116)=2.52, p=0.12$. The threeway interaction between retrieval trial, age and retrieval condition also failed to reach significance, $F(1,36)=2.01$, $p=0.16$. These results show that both younger and older adults benefit from repeated collaborative recall trials beyond that of repeated individual recall trials. These results also show that such collaboration benefits can last up to 1 week. This is the first demonstration of collaboration benefits on later individual recall at delays as long as 1 week in both younger and older adults. A trend towards greater collaboration benefits in younger compared to older adults after a short delay and similar collaboration benefit in younger and older adults after a long delay is consistent with the idea that younger adults benefit more from re-exposure than older adults while older adults are better able to use the recall of other group members as cross-cues during collaborative recall. However, future studies will determine if this interaction is in fact reliable.

\section{Hypermnesia}

The proportions of hypermnesia across recall trials in younger and older adults are displayed in Table 2. The presence of cross-cuing during repeated collaborative recall trials (Recall 1 to Recall 2) was assessed in a betweensubject ANOVA with age (Young versus Old) and retrieval

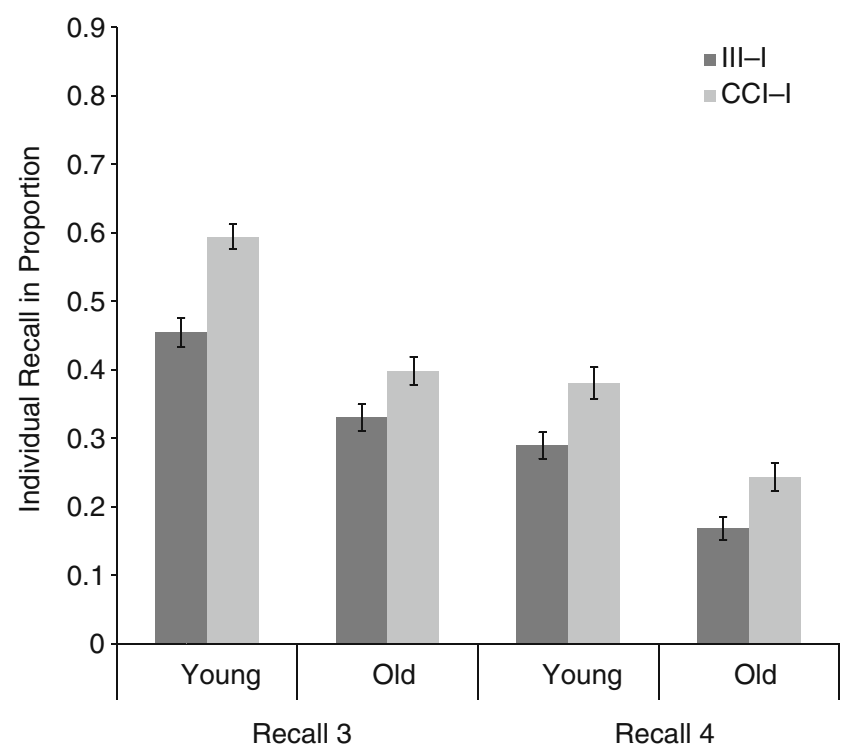

Fig. 2 Mean proportion of correct individual recall in each age group and in each retrieval sequence condition during Recall 3 and Recall 4 
Table 2 Proportions of hypermnesia and recovery across recall trials in each age group and in each retrieval sequence condition

\begin{tabular}{|c|c|c|c|c|c|c|}
\hline \multirow[t]{2}{*}{ Condition } & \multicolumn{2}{|c|}{ Recall 1 to Recall 2} & \multicolumn{2}{|c|}{ Recall 2 to Recall 3} & \multicolumn{2}{|c|}{ Recall 3 to Recall 4} \\
\hline & Younger & Older & Younger & Older & Younger & Older \\
\hline \multicolumn{7}{|c|}{ III-I (Individual) } \\
\hline Hypermnesia & 0.04 & 0.01 & 0.03 & 0.01 & -0.17 & -0.16 \\
\hline Recovery & 0.08 & 0.05 & 0.07 & 0.05 & 0.03 & 0.02 \\
\hline \multicolumn{7}{|c|}{ CCI-I (Collaborative) } \\
\hline Hypermnesia & 0.06 & 0.05 & -0.10 & -0.14 & -0.21 & -0.16 \\
\hline Recovery & 0.10 & 0.09 & 0.03 & 0.03 & 0.03 & 0.04 \\
\hline
\end{tabular}

condition (Collaborative or Individual) as the betweensubjects measures. We assumed that cross-cuing would be present if hypermnesia from Recall 1 to Recall 2 was greater during repeated collaborative recall than repeated individual recall (Blumen \& Rajaram, 2008; Weldon \& Bellinger, 1997; Meudell et al., 1992, 1995). There was a main effect of age $F(1,116)=6.52, p<0.05, \eta p^{2}=0.05$ reflecting greater hypermnesia from Recall 1 to Recall 2 in younger compared to older adults. There was also a main effect of retrieval condition, $F(1,116)=6.11, p<0.05, \eta^{2}=$ 0.05 , reflecting greater hypermnesia during repeated collaborative recall than during repeated individual recall. The interaction between age and retrieval condition was not significant, $F(1,116)=0.68, p>0.05$. These results suggest that during repeated collaborative recall trials, the recall of other group members triggered recall of new information that would not be available to individuals if they had been recalling alone (i.e. cross-cuing). Although hypermnesia in general was greater in younger than older adults, cross-cuing did not differ between age groups. These findings are small but reliable, replicate previous findings in younger adults (Blumen \& Rajaram, 2008) and extend them to older adults.

\section{Recovery}

The recovery measure was used to examine whether recovery would be greater shortly following collaborative than individual recall trials (indicating a recovery from retrieval disruption) and whether recovery would continue to be greater following collaborative than individual recall trials after a 1-week delay (indicating a release from SSRIF). To this end, recovery as a function of age and retrieval trial was assessed in a repeated measure ANOVA with recall trial (Recall 2 to Recall 3 and Recall 3 to Recall 4) as the within-subjects variable, and age (Younger and Older) and retrieval condition (Collaborative or Individual) as between-subjects variables. The proportions of recovery in younger and older adults are displayed in Table 2 . There was a main effect of recall trial, $F(1,116)=9.59, p<0.01, \eta p^{2}=$ 0.08 , indicating greater levels of recovery from Recall 2 to Recall 3 than Recall 3 to Recall 4. The main effect of age was not significant, $F(1,116)=2.08, p=15$ and recall trial did not interact with age $F(1,116)=1.43, p>0.05$. There was, however, a main effect of condition, $F(1,116)=7.10$, $p<0.01, \eta \mathrm{p}^{2}=0.06$ and an interaction between recall trial and condition, $F(1,116)=14.70, \eta \mathrm{p}^{2}=0.11$, such that recovery from Recall 2 to Recall 3 was greater in the III-I condition compared to the CCI-I $(t(118)=4.67, p<0.001)$, but recovery from Recall 3 to Recall 4 was similar in the IIII condition and the CCI-I condition $(t(118)=-0.90, p>$ $0.05)$. There was also an interaction between age and condition, $F(1,116)=8.56, \eta \mathrm{p}^{2}=0.07$, such that recovery (collapsed across Recall 2 to 3 and Recall 3 to 4 ) in younger adults was greater in the III-I condition than in the CCI-I condition $(\mathrm{t}(58)=3.57, p<0.01)$, but in older adults recovery was similar in the III-I condition and the CCI-I condition $(t(58)=0.21, p>0.05)$. Finally, there was no three-way interaction between retrieval trial, age and retrieval condition, $F(1,116)=0.04, p>0.05$. These recovery analyses suggest that recovery shortly following repeated individual recall is greater than shortly following repeated collaborative recall, but that this difference dissipates after a longer delay. These recovery analyses are inconsistent with the prediction that recovery will be greater shortly following repeated collaborative recall than repeated individual recall (indicating a recovery from retrieval disruption) and the prediction that recovery will be greater a week following repeated collaborative recall than repeated individual recall (indicating a release from SS-RIF). Instead, these analyses point to further examinations of how reexposure and cross-cuing influence later individual memory.

\section{General discussion}

Very little is known about how prior group collaboration modulates later individual memory in younger and older adults. The key finding of our study was that repeated collaborative recall trials generate short-term and long-term benefits on later individual recall in both younger and older adults. More specifically, both younger and older adults benefitted from repeated collaborative recall trials to a 
greater extent than repeated individual recall trials, and such collaboration benefits lasted up to 1 week following the initial study and recall phase. This is the first demonstration of collaboration benefits on later individual recall at delays as long as 1 week in both younger and older adults, although long-term collaboration benefits on later individual recognition performance in younger adults have been reported previously (Rajaram \& Pereira-Pasarin, 2007). The short-term collaboration benefits observed in our study are a function of both re-exposure and crosscuing, because the net increase in recall across recall trials was greater during repeated collaborative recall trials than during repeated individual recall trials in both younger and older adults. The long-term collaboration benefits observed in our study more likely are the results of cross-cuing, as cross-cuing is more comparable to additional retrieval opportunities and has been shown to be critical for longterm retention (e.g., Roediger \& Karpicke, 2006). However, designs that simultaneously control or manipulate reexposure and cross-cuing are required to provide direct support for this proposition. A trend towards greater shortterm collaboration benefits in younger rather than older adults and similar long-term collaboration benefits in younger and older adults was also observed in this study. Future studies are needed to confirm whether this trend is indeed reliable and whether they support the idea that younger adults benefit more from re-exposure than older adults while older adults are better able to use the recall of others group members as cross-cues during collaborative recall (Craik, Byrd \& Swanson, 1987; Craik \& McDowd, 1987).

Our study also showed that the negative effects of collaboration on group memory do not differ as a function of age or retrieval trials. Similar levels of collaborative inhibition in younger and older adults replicate previous research (Ross et al., 2008; Meade \& Roediger, 2009) and extend them to repeated collaborative recall trials. Moreover, our study failed to find evidence for greater recovery shortly following repeated collaborative recall trials than repeated individual recall trials (indicative of recovery from retrieval disruption), and greater recovery a week following repeated collaborative recall trials than repeated individual recall trials (indicative of a release from SS-RIF). These findings can potentially be interpreted in two ways. First, it is possible that retrieval disruption and or SS-RIF continue to reduce later individual memory at a shorter and longer delay. However, previous research suggests that this is not the case (Finlay et al., 2000; MacLeod \& Macrae, 2001). Second, it is possible that both short-term and long-term collaboration benefits on later individuals are independent upon the release from retrieval disruption and or SS-RIF. While the former point to more specific examinations of how retrieval disruption and or SS-RIF operate during collaborative recall, the latter point to more specific examinations of how re-exposure and cross-cuing influence later individual memory over time. In any case, designs that simultaneously, control or manipulate retrieval disruption and SS-RIF, or re-exposure and cross-cuing, are required to address these issues.

Taken together, the results of our study suggest that repeated collaborative recall trials enhance later individual recall after a short delay and after a long delay, in both younger and older adults. These initial findings are promising but point to more specific examinations of how re-exposure and cross-cuing influence later individual recall over time. Future studies should also aim to examine if prior group collaboration can benefit later individual memory in realworld settings, with stimuli relevant to everyday life, and across different retrieval tasks (for a retrieval task comparison in younger adults see Blumen \& Rajaram, 2009).

Acknowledgements We would like to thank Lauren Altamura, Zoltan Apa and Preeti Sunderaraman for their research assistance and Brian C. Rakitin for his statistical advice.

\section{References}

Anderson, M. C. (2005). The role of inhibitory control in forgetting unwanted memories: A consideration of three methods. In C. MacLeod \& B. Uttl (Eds.), Dynamic cognitive processes (pp. 159-190). Tokyo: Springer-Verlag. doi:10.1007/ 4-431-27431-6 8.

Anderson, M. C., Bjork, R. A., \& Bjork, E. L. (1994). Remembering can cause forgetting: Retrieval dynamics in long-term memory. Journal of Experimental Psychology. Learning, Memory, and Cognition, 20, 1063-1087. doi:10.1037/0278-7393.20.5.1063.

Basden, B. H., Basden, D. R., Bryner, S., \& Thomas, R. L., III. (1997). A comparison of group and individual remembering: Does collaboration disrupt retrieval strategies? Journal of Experimental Psychology. Learning, Memory, and Cognition, 23(5), 1176-1191. doi:10.1037/0278-7393.23.5.1176.

Blumen, H. M., \& Rajaram, S. (2008). Influence of re-exposure and retrieval disruption during group collaboration on later individual recall. Memory, 16(3), 231-244. doi:10.1080/09658 210701804495.

Blumen, H. M., \& Rajaram, S. (2009). Effects of repeated collaborative retrieval on individual memory vary as a function of recall versus recognition tasks. Memory, 17(8), 840-846. doi:10.1080/ 09658210903266931.

Clark, J. M., \& Paivio, A. (2004). Extensions of the Paivio, Yuille and Madigan (1968) norms. Behavior Research Methods, Instruments \& Computers, 36(3), 371-383.

Coman, A., Manier, D., \& Hirst, W. (2009). Forgetting the unforgettable through conversation: Socially shared retrievalinduced forgetting of September 11 memories. Psychological Science, 20(5), 627-633. doi:10.1111/j.1467-9280.2009.02343.x.

Craik, F. I. M., Byrd, M., \& Swanson, J. M. (1987). Patterns of memory loss in three elderly samples. Psychology and Aging, 2, 79-86. doi:10.1037/0882-7974.2.1.79.

Craik, F. I. M., \& McDowd, J. M. (1987). Age differences in recall and recognition. Journal of Experimental Psychology: Learning, 
Memory, and Cognition, 13, 474-479. doi:10.1037/02787393.13.3.474.

Cuc, A., Koppel, J., \& Hirst, W. (2007). Silence is not golden: A case for socially-shared retrieval-induced forgetting. Psychological Science, 18(9), 727-733. doi:10.1111/j.14679280.2007.01967.x.

Finlay, F., Hitch, G. J., \& Meudell, P. R. (2000). Mutual inhibition in collaborative recall: Evidence for a retrieval-based account. Journal of Experimental Psychology. Learning, Memory, and Cognition, 26(6), 1556-1567. doi:10.1037/0278-7393.26.6.1556.

Kelley, M. R., \& Wright, D. B. (2010). Obtaining representative nominal groups. Behavior Research Methods, 42(1), 36-41. doi:10.3758/BRM.42.1.36.

MacLeod, M. D., \& Macrae, C. N. (2001). Gone but not forgotten: The transient nature of retrieval-induced forgetting. Psychological Science, 12(2), 148-152. doi:10.1111/1467-9280.00325.

Mattis, S. (1976). Mental status examination for organic mental syndrome in the elderly patient. In L. Bellak \& T. B. Karasu (Eds.), Geriatric psychiatry (pp. 77-121). New York: Grune \& Stratton.

Meade, M. L., \& Roediger, H. L., III. (2009). Age differences in collaborative memory: The role of retrieval manipulations. Memory \& Cognition, 37(7), 962-975. doi:10.3758/ MC.37.7.962.

Meudell, P. R., Hitch, G. J., \& Boyle, M. M. (1995). Collaboration in recall: Do pairs of people cross-cue each other to produce new memories? The Quarterly Journal of Experimental Psychology. A: Human Experimental Psychology, 48(1), 141-152.

Meudell, P. R., Hitch, G. J., \& Kirby, P. (1992). Are two heads better than one? Experimental investigations of the social facilitation of memory. Applied Cognitive Psychology, 6(6), 525-543. doi:10.1002/acp.2350060606.

Payne, D. G. (1987). Hypermnesia and recovery in recall: A historical and empirical review. Psychological Bulletin, 101(1), 5-27. doi:10.1037/0033-2909.101.1.5.

Rajaram, S., \& Pereira-Pasarin, L. P. (2007). Collaboration can improve individual recognition memory: Evidence from immediate and delayed tests. Psychonomic Bulletin \& Review, 14(1), 95-100.

Roediger, H. L., III, \& Karpicke, J. D. (2006). Test-enhanced learning: Taking memory tests improves long-term retention. Psychological Science, 17(3), 249-255. doi:10.1111/j.1467-9280.2006.01693.x.

Ross, M., Spencer, S. J., Blatz, C. W., \& Restorick, E. (2008). Collaboration reduces the frequency of false memories in older and younger adults. Psychology and Aging, 23(1), 85-92. doi:10.1037/0882-7974.23.1.85.

Stone, C. B., Barnier, A. J., Sutton, J., \& Hirst, W. (2010). Building consensus about the past: Schema consistency and convergence in socially shared retrieval-induced forgetting. Memory, 18(2), 170-184. doi:10.1080/09658210903159003.

Weldon, M. S., \& Bellinger, K. D. (1997). Collective memory: Collaborative and individual processes in remembering. Journal of Experimental Psychology. Learning, Memory, and Cognition, 23(5), 1160-1175. doi:10.1037/0278-7393.23.5.1160.

Wright, D. B. (2007). Calculating nominal group statistics in collaboration studies. Behavior Research Methods, 39, 460 470.

Wright, D. B., \& Klumpp, A. (2004). Collaborative inhibition is due to the product, not the process, of recalling in groups. Psychonomic Bulletin \& Review, 11(6), 1080-1083. 